\title{
2-Cyano-2-phenylpropanoic Acid Triggers the Back and Forth Motions of an Acid-Base-Operated Paramagnetic Molecular Switch
}

\author{
Paola Franchi, ${ }^{\dagger}$ Cecilia Poderi, $^{\dagger}$ Elisabetta Mezzina, $^{\dagger}$ Chiara Biagini, $^{\ddagger}$, Stefano Di Stefano, $^{*}+, \S \odot$ \\ and Marco Lucarini*, ${ }^{*} \odot$ \\ ${ }^{\dagger}$ Dipartimento di Chimica “G. Ciamician”, Università di Bologna, Via San Giacomo 11, I-40126 Bologna, Italy \\ ${ }^{\ddagger}$ Dipartimento di Chimica, Università di Roma La Sapienza, P.le A. Moro, 5, I-00185 Rome, Italy \\ ${ }^{\S}$ Sezione Meccanismi di Reazione, Istituto CNR per i Sistemi Biologici (ISB-CNR), P.le A. Moro 5, I-00185 Roma, Italy
}

ABSTRACT: The back and forth motions of a crown-ether based wheel along the axis of a bistable rotaxane are triggered by the decarboxylation of 2-cyano-2-phenylpropanoic acid and detected by the oscillation of the EPR nitrogen splitting of a dialkyl nitroxide function mounted within the macrocyclic ring. When the $p$-Cl derivative of the acid is used, back and forth motions are accelerated. Conversely, with $p-\mathrm{CH}_{3}$ and $p-\mathrm{OCH}_{3}$ derivatives, the back motion is strongly inhibited by the insurgence of collateral radical reactions.

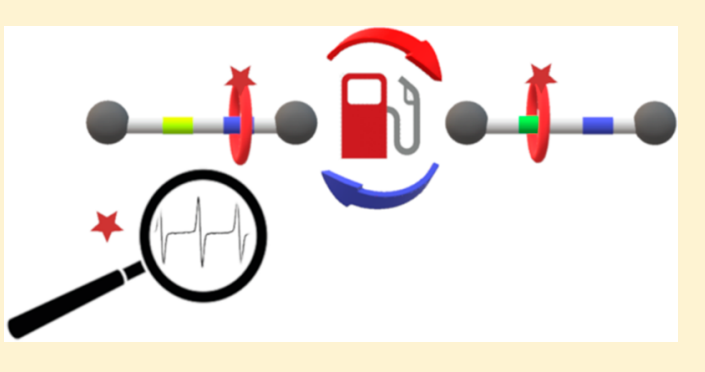

T $\mathrm{n}$ the course of the last decades, supramolecular chemists have designed and realized a large variety of molecular machines (switches and motors), which undergo large amplitude motions under the influence of external stimuli. ${ }^{1}$ Mechanically interlocked molecules (MIMs), namely catenanes and rotaxanes, ${ }^{2,3}$ which shift among two or more states due to the action of chemical or radiative stimuli, are well established examples. Most of them require subsequent additions of chemical reagents or irradiations with light at different wavelengths to complete a whole cycle of motions (from the initial state to another state or a sequence of other states, and back again to the initial state). ${ }^{1 a}$ Instead, only in a handful of cases are the molecular machines based on MIMs able to exploit just one radiative ${ }^{4}$ or chemical $^{5}$ stimulus to pass from an initial state to one or more intermediate states and return to the initial state without necessity of a counterstimulus.

One impressive example is a [2]-catenane described by Leigh and co-workers. ${ }^{5 b}$ Here, one of the macrocycle composing the catenane carries out unidirectional and autonomous cycles of motion around the other macrocycle, being fed on just one only stimulus.

Another example, developed in our laboratory, is based on a Sauvage-type catenane, ${ }^{5 \mathrm{a}, \mathrm{d}, \mathrm{f}}$ which can move back and forth among a state $\mathrm{A}$ and two subsequent states $\mathrm{B}\left(\mathrm{B}^{\prime}\right.$ and $\left.\mathrm{B}^{\prime \prime}\right)$, when 1 molar equiv of acid 1 (2-phenyl-2-cyanopropanoic acid) is added (see Scheme 1).

This was the first case in which a molecular machine, namely a switch, performs a whole cycle of motion $(\mathrm{A} \rightarrow \mathrm{B} \rightarrow \mathrm{A})$ with no need of any counter-stimulus. Coupling of the $\mathrm{A} \rightarrow \mathrm{B} \rightarrow \mathrm{A}$ transitions to the step-by-step decarboxylation of reactant $\mathbf{1}$ to product 2 (2-phenyl-2-cyanopropane) is the key point of the system (Scheme 1).

In addition, Schmittel and co-workers exploited acid $\mathbf{1}$ to promote the back and forth motions of an acid-base operated
Scheme 1. A $\rightarrow$ B $\rightarrow$ A Cycle of Motion Coupled to the Decarboxylation of Acid $1^{a}$

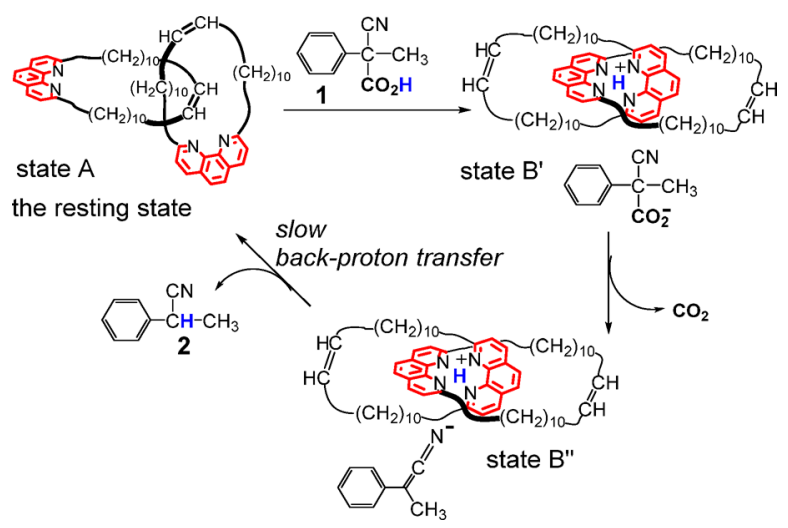

${ }^{a}$ Fast and quantitative proton transfer (step $\mathrm{A} \rightarrow \mathrm{B}^{\prime}$ ) causes the two phenanthroline subunits to approach one another in order to share the proton. Decarboxylation $\left(\mathrm{B}^{\prime} \rightarrow \mathrm{B}^{\prime \prime}\right)$, and following, slow backproton transfer restore the resting state of the catenane $\left(B^{\prime \prime} \rightarrow A\right)$.

[2]-rotaxane switch, $^{\text {Se }}$ while Leigh and co-workers, taking inspiration from this acid, took advantage of the decarboxylation of trichloroacetic acid to supply energy to a [2]catenane based motor where one of the macrocycles performs oriented $360^{\circ}$ circumrotations around the other. ${ }^{5 c}$

Here, we report on the use of compound $\mathbf{1}$ and derivatives 3-5 (Scheme 2) to trigger the back and forth motions of a recently proposed bistable nitroxide-containing [2]-rotaxane, $\mathbf{6 H} \cdot\left(\mathrm{PF}_{6}\right)_{3}$, which is able to change coconformation under an acid-base stimulus. ${ }^{6}$ The [2]-rotaxane architecture comprises

Received: April 29, 2019

Published: June 17, 2019 
Scheme 2

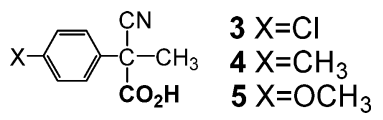

a dialkyl nitroxide functionality inserted in a crown ether-like ring interlocked with a dumbbell component that possesses two different recognition sites, namely, a dialkylammonium $\left(\mathrm{NH}_{2}^{+}\right)$and a 4,4'-bipyridinium $\left(\mathrm{BPY}^{2+}\right)$ unit (Scheme 3). In $\mathbf{6 H} \cdot\left(\mathrm{PF}_{6}\right)_{3}$, the macrocycle is not significantly interacting with the $\mathrm{BPY}^{2+}$ unit, consistent with it being localized predominantly over the ammonium site of the thread.

Scheme 3. Switching Process between $6 \mathrm{H} \cdot\left(\mathrm{PF}_{6}\right)_{3}$ and $6 \cdot$ $\left(\mathrm{PF}_{6}\right)_{2}$

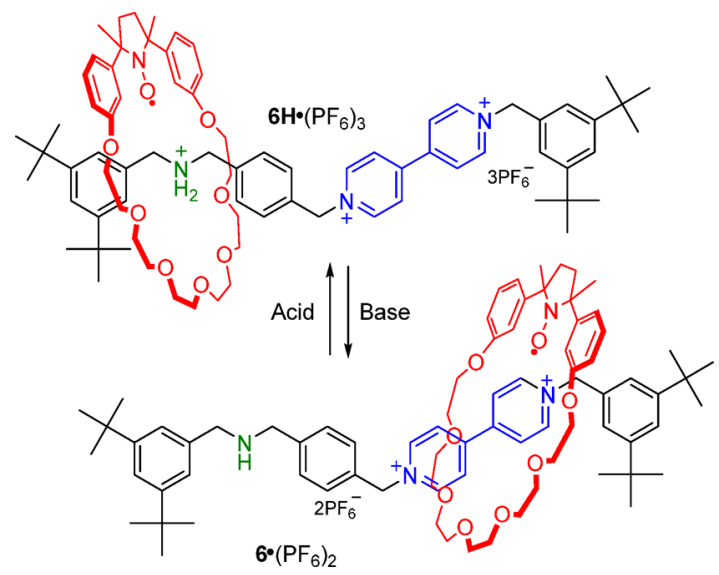

Shuttling of the paramagnetic macrocycle toward the secondary $\mathrm{BPY}^{2+}$ station (Scheme 3 ) is triggered by treating rotaxane $6 \mathrm{H} \cdot\left(\mathrm{PF}_{6}\right)_{3}$ with the non-nucleophilic Hünig's base, diisopropylethylamine $\left(i \operatorname{Pr}_{2} \mathrm{EtN}\right)$, which is strong enough to deprotonate the $\mathrm{NH}_{2}{ }^{+}$center. ${ }^{7 a}$ Addition of trifluoroacetic acid causes the return back movement of the ring onto the preferred ammonium site. Owing to the paramagnetic nature of the rotaxane, the back and forth motion could be well detected by EPR spectroscopy.

In particular, we demonstrated that when the macrocycle predominantly encircles the ammonium station the nitrogen splitting value $a_{\mathrm{N}}$ is $14.39 \mathrm{G}$ and noticeably increases to 14.82 $\mathrm{G}$ after addition of 1 equiv of $i \operatorname{Pr}_{2} \mathrm{EtN}\left(\Delta a_{\mathrm{N}}=+0.43 \mathrm{G}\right.$, see Figure 1).

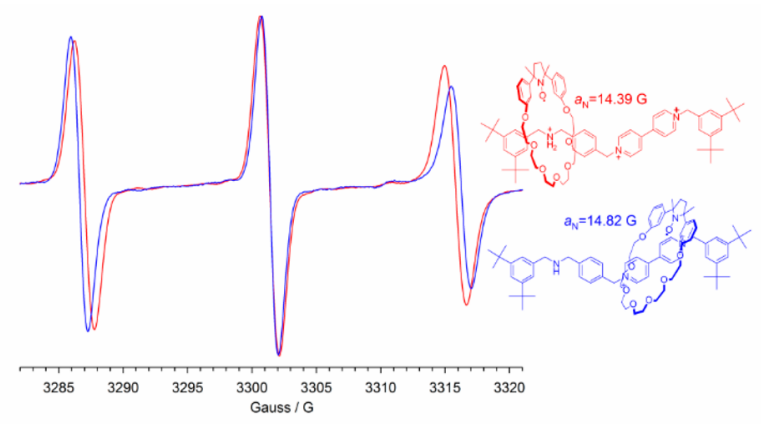

Figure 1. EPR spectra of tricharged $(6 \mathrm{H})$ and dicharged (6) rotaxanes in $\mathrm{CH}_{2} \mathrm{Cl}_{2}$.
With the aim of realizing one-only stimulus supplied back and forth motions of the paramagnetic wheel in rotaxane $6 \mathbf{H}$. $\left(\mathrm{PF}_{6}\right)_{3}$, we decided to employ acid 1 to drive the switchable molecular shuttle in $\mathbf{6 H} \cdot\left(\mathrm{PF}_{6}\right)_{3}$.

To investigate the acid $\mathbf{1}$-driven reversible cycle, we started from deprotonated rotaxane $6 \cdot\left(\mathrm{PF}_{6}\right)_{2}$, in which the preferred coconformation is that of ring-complexed viologen unit. This was accomplished by adding 1 molar equiv of $i \operatorname{Pr}_{2} \mathrm{EtN}$ to $6 \mathrm{H}$. $\left(\mathrm{PF}_{6}\right)_{3}$. Treatment of the resulting rotaxane $6 \cdot\left(\mathrm{PF}_{6}\right)_{2}$ with 1 molar equiv of acid 1 in $\mathrm{CH}_{2} \mathrm{Cl}_{2}$ causes a drastic change in the EPR spectrum within a few seconds with concomitant formation of $\left[6 \mathbf{H} \cdot\left(\mathrm{PF}_{6}\right)_{2}\right]^{+} \cdot \mathrm{RCO}_{2}^{-}$. Spectra were measured at constant time intervals after addition of 1 (see Figure 2). Over

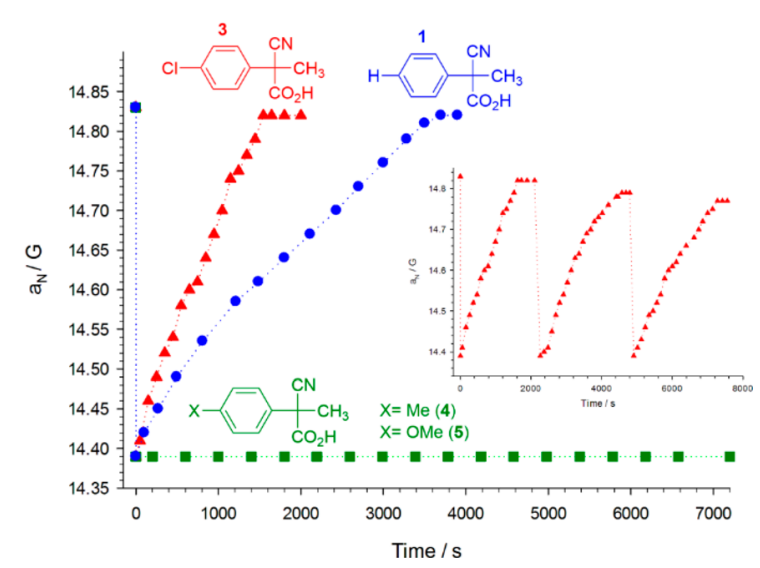

Figure 2. Time variation of $a_{\mathrm{N}}$ after the addition of 1 molar equiv of different acids $(1,3-5)$ to $6 \cdot\left(\mathrm{PF}_{6}\right)_{2}$ in $\mathrm{CH}_{2} \mathrm{Cl}_{2}$. Inset: $a_{\mathrm{N}}$ variation in rotaxane 6 as a function of sequential fuel 3 additions.

the course of ca. $60 \mathrm{~min}$, the nitrogen splitting constant increases constantly, and full back transformation to $6 \cdot\left(\mathrm{PF}_{6}\right)_{2}$ is clearly proved by the appearance of nitrogen splitting original value corresponding to the location of the macrocycle on the $\mathrm{BPY}^{2+}$ unit (see Figure 2). ${ }^{6}$

In the course of several fueled cycles (as an example, see Figure 2, inset), each ignited by addition of 1 molar equiv of acid, the nitrogen splitting drops rapidly after each addition and recuperates, slightly lower to the original level over ca. the same time, as defined by state A.

It has already been shown that the rate of back proton transfer, which in the absence of competitive processes determines the rate of the overall process, is markedly affected by the nature of the para-substituent in the aromatic ring of 1. ${ }^{5 \mathrm{~d}}$ Thus, we decided to monitor by EPR the back and forth motions of the paramagnetic wheel in the presence of some representative acid $\mathbf{1}$ derivatives containing electron-withdrawing $(\mathrm{Cl})$ and electron-donating $(\mathrm{Me}, \mathrm{OMe})$ parasubstituents. As expected, with the chlorinated derivative 3 the time required to complete a full cycle was significant faster compared to that of the unsubstituted derivative (see Figure 2 ). Unexpected result were instead observed in the presence of acids containing an electron donating group (4 and 5 ). While addition of 1 molar equiv of 4 or 5 to rotaxane $6 \cdot\left(\mathrm{PF}_{6}\right)_{2}$ in $\mathrm{CH}_{2} \mathrm{Cl}_{2}$ causes a decrease in the EPR $a_{\mathrm{N}}$ value $(14.39 \mathrm{G})$ within a few seconds as expected for the concomitant formation of the protonated tricharged form of the rotaxane, no recovery of the initial value of nitrogen splitting $(14.82 \mathrm{G})$ was observed with both fuels even after several hours upon mixing of the reagents (see Figure 2). 
Scheme 4. Switching Motions of Rotaxane 6•(PF $)_{2}$ Triggered by Acid $1^{a}$

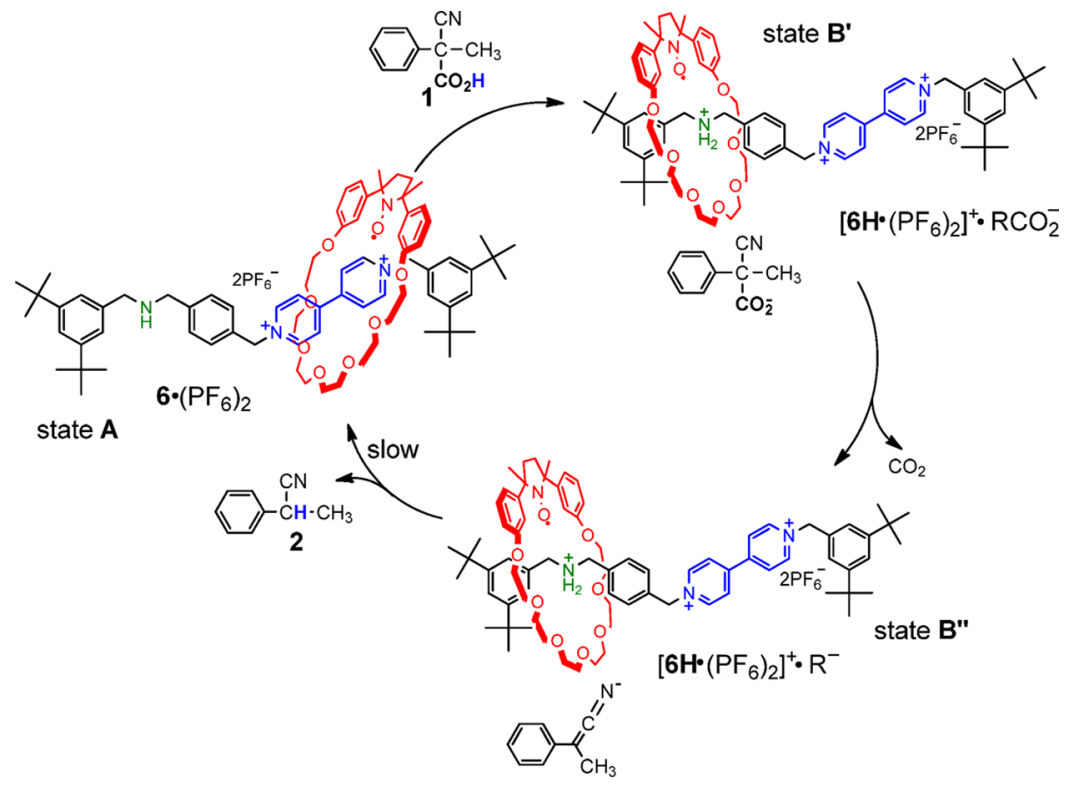

${ }^{a}$ Fast and quantitative proton transfer (step $\mathbf{A} \rightarrow \mathbf{B}^{\prime}$ ) causes the movement of the wheel onto the ammonium site of the dumbbell. Loss of $\mathrm{CO}_{2}$ $\left(\right.$ step $\left.B^{\prime} \rightarrow B^{\prime \prime}\right)$ followed by slow back proton transfer from $\left[6 \mathbf{H} \cdot\left(\mathrm{PF}_{6}\right)_{2}\right]^{+}$to its counteranion $\left(\mathrm{R}^{-}\right)$restores the position of the ring to the bipyridinium station (step $\mathbf{B}^{\prime \prime} \rightarrow \mathbf{A}$ ).

Scheme 5. Competitive SET Mechanism (Only Active when $\mathrm{X}=\mathrm{CH}_{3}$ or $\mathrm{OCH}_{3}$ )

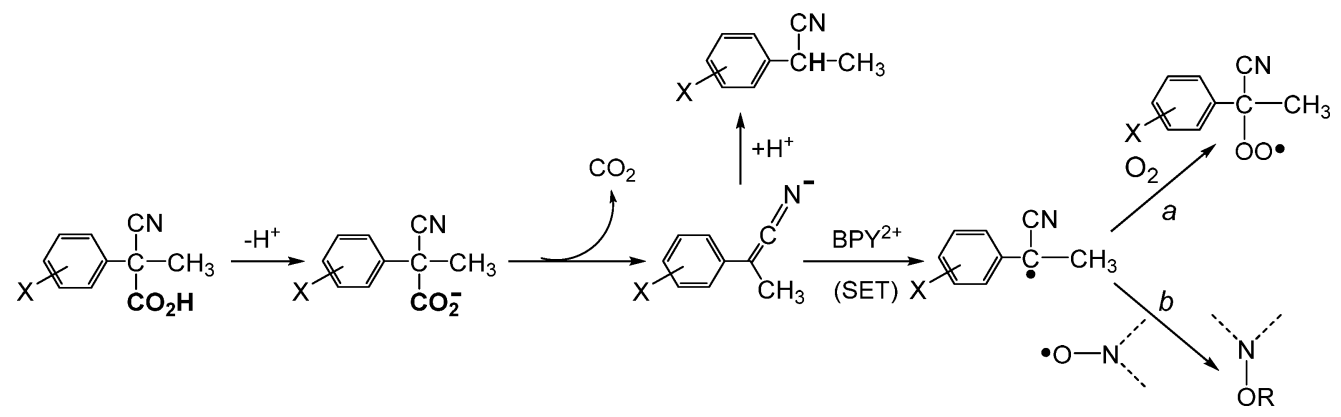

Slow return to initial state A (Scheme 1) in the presence of 4 and 5 has been already demonstrated for a Sauvage-type catenane. ${ }^{5 \mathrm{~d}}$ Thus, failure in the retrieval of state $\mathbf{A}$ observed in the present case suggests that some alternative processes should compete with the final back proton transfer within ionpair $\left[6 \mathrm{H} \cdot\left(\mathrm{PF}_{6}\right)_{2}\right]^{+} \cdot\left(\mathrm{R}^{-}\right)$from $6 \mathrm{H}$ to benzyl anion $\left(\mathrm{R}^{-}\right)$(see Scheme 4).

Benzyl anions can lose one electron to afford neutral benzyl radicals, and this process is much more feasible when they contain an electron-donating substituent in the para-position as revealed by the corresponding half-wave potentials $\left(E_{1 / 2}\right)$ for the electrochemical reduction of 4-substituted benzyl radicals. ${ }^{8}$ Since the rotaxane thread contains the $1,1^{\prime}$-dialkyl$4,4^{\prime}$-bipyridinium unit which is known to undergo monoelectronic reduction at relatively low reduction potential ( -0.42 vs SCE in ACN), it seems reasonable to hypothesize an electron transfer from the benzyl anion to $\mathrm{BPY}^{2+}$ unit as an alternative process to deprotonation of the rotaxane (see Scheme 5). The single-electron transfer (SET) process leads to the BPY radical cation and a benzyl radical which in turn is trapped by oxygen to afford the corresponding peroxyl radical (Scheme 5, path a).

Since this process is expected to consume molecular oxygen, we checked the feasibility of this path by repeating the stimulus-driven transformation in a closed tube by following the oxygen consumption during an entire cycle. The change of oxygen concentration after the addition of 4 or 5 within the closed tube was determined by following the evolution of the three lines EPR spectrum of the nitroxidic rotaxane. Actually the width of these lines is related to the concentration of oxygen, which produces a strong broadening of the EPR spectral lines of radicals due to Heisenberg spin exchange. ${ }^{9,10}$

Another spectral parameter, sensitive to the oxygen concentration, but much easier to measure than the width, is the peak to peak height of the EPR line, $I$. This is proportional to both the radical concentration and the reciprocal of line width. In Figure 3, the dependence of the intensity of the central line of protonated tricharged form of the rotaxane on time after addition of acid $\mathbf{4}$ is shown. At the beginning of the reaction the intensity of the EPR lines increases since the benzyl radicals produced in the SET step react with $\mathrm{O}_{2}$ (path a). ${ }^{11}$ Molecular oxygen is therefore consumed in the reaction vessel, the width of the EPR lines due to the nitroxide decreases, and their height increases. Only when most of the oxygen has been consumed do the benzyl radicals begin to react with the protonated rotaxane $\mathbf{6 H}$ (Scheme 5 , path $\mathrm{b}$ ), leading to the degradation of the paramagnetic nitroxidic probe and the concomitant decrease of the EPR signal intensities. It 


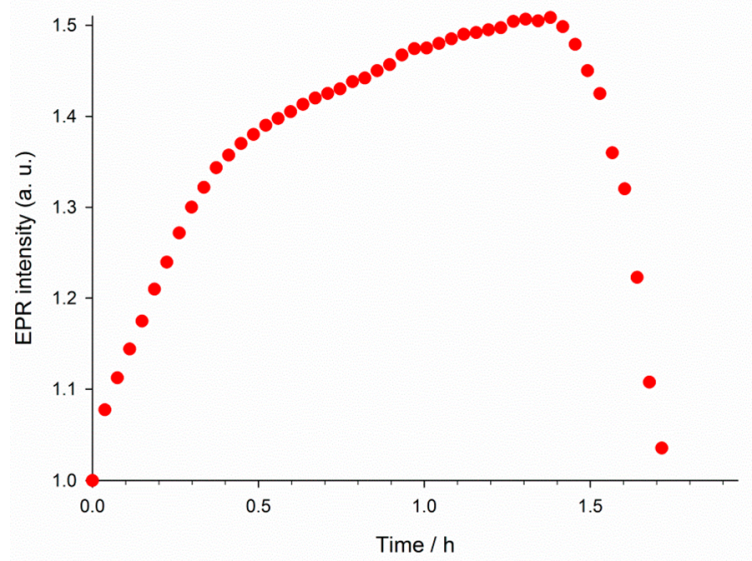

Figure 3. Circles show the experimental time dependence of the height of the central line $(I)$ of the EPR spectrum of the tricharged rotaxane $6 \mathrm{H}$ after addition of 1 molar equiv of 4 at room temperature.

should be noted that no evidence of oxygen consumption during a complete cycle was observed with fuels 1 and 3 (data not shown).

In conclusion, the reported paramagnetic rotaxane is able to perform back and forth switching motions in the presence of activated carboxylic acids. Different from all previously reported cases for which the back and forth motions of switches or motors were monitored by common spectroscopic tools (NMR, spectrophotometry, or spectrofluorimetry), in this case EPR spectroscopy was employed for the first time to demonstrate the movement. This report shows that, apart from limitations dictated by the peculiar red-ox features of this specific system (electron donating substituents at the para position of the aromatic ring of the fuel are not tolerated), 2cyano-2-phenylpropanoic acid and its derivatives can be used to trigger the back and forth motions of a complex molecular machine incorporating a radical center and to modulate its magnetic properties.

\section{EXPERIMENTAL SECTION}

Rotaxane $\mathbf{6 H} \cdot\left(\mathrm{PF}_{6}\right)_{3}$ was prepared as previously reported. ${ }^{6}$ Fuels $\mathbf{1 - 4}$ were available from previous investigation. ${ }^{5 \mathrm{~d}}$

EPR Measurements. EPR spectra have been recorded on a Bruker-ELEXYS spectrometer by using the following instrument settings: microwave power $0.79 \mathrm{~mW}$, modulation amplitude $0.04 \mathrm{mT}$, modulation frequency $100 \mathrm{kHz}$, scan time $180 \mathrm{~s}, 2 \mathrm{~K}$ data points. The hyperfine splittings were determined by computer simulation using a Monte Carlo minimization procedure. ${ }^{12}$

EPR Kinetic Studies for the Fuel-Driven Reversible Cycle. A solution of $6 \mathbf{H} \cdot\left(\mathrm{PF}_{6}\right)_{3}(0.1 \mathrm{mM})$ and diisopropylethylamine amine $(0.1 \mathrm{mM})$ is introduced (ca. $50 \mu \mathrm{L})$ into a capillary tube with an internal diameter of ca. $1.85 \mathrm{~mm}$. The sample is then introduced in the EPR cavity, and an EPR spectrum is recorded to verify the complete deprotonation of $6 \mathrm{H} \cdot\left(\mathrm{PF}_{6}\right)_{3}$. Subsequently, 1 molar equiv of the proper fuel is added, and EPR spectra are measured at constant time intervals. Oxygen consumption studies were performed by a similar procedure. In this case, the sample was closed by introducing into the sample tube a second capillary tube (external diameter of $1.60 \mathrm{~mm}$ ) sealed at one end and leaving very little dead volume space. The decrease of the nitroxide concentration and the oxygen uptake were followed by EPR spectroscopy as described in the paper.

\section{AUTHOR INFORMATION}

\section{Corresponding Authors}

*E-mail: marco.lucarini@unibo.it.
*E-mail: stefano.distefano@uniroma1.it.

ORCID

Stefano Di Stefano: 0000-0002-6742-0988

Marco Lucarini: 0000-0002-8978-4707

Notes

The authors declare no competing financial interest.

\section{ACKNOWLEDGMENTS}

This work was supported by the University of Bologna and the University of Roma La Sapienza (Progetti di Ricerca Grandi 2018, prot. n. RG1181641DCAAC4E).

\section{REFERENCES}

(1) (a) Erbaş-Çakmak, S.; Leigh, D. A.; McTernan, C. T.; Nussbaumer, A. L. Artificial Molecular Machine. Chem. Rev. 2015, 115, 10081-10206. (b) Kassem, S.; van Leeuwen, T.; Lubbe, A. S.; Wilson, M. R.; Feringa, B. L.; Leigh, D. A. Artificial Molecular Motors. Chem. Soc. Rev. 2017, 46, 2592-2621. (c) Baroncini, M.; Casimiro, L.; de Vet, C.; Groppi, J.; Silvi, S.; Credi, A. Making and Operating Molecular Machines: A Multidisciplinary Challenge. ChemistryOpen 2018, 7, 169-179.

(2) Selected examples: (a) Bissell, R. A.; Cordova, E.; Kaifer, A. E.; Stoddart, J. F. A Chemically and Electrochemically Switchable Molecular Shuttle. Nature 1994, 369, 133-137. (b) Badjic, J. D.; Balzani, V.; Credi, A.; Silvi, S.; Stoddart, J. F. A Molecular Elevator. Science 2004, 303, 1845-1849. (c) Blanco, V.; Leigh, D. A.; Marcos, V.; Morales-Serna, J. A.; Nussbaumer, A. L. A Switchable [2]Rotaxane Asymmetric Organocatalyst That Utilizes an Acyclic Chiral Secondary Amine. J. Am. Chem. Soc. 2014, 136, 4905-4908. (d) Armaroli, N.; Balzani, V.; Collin, J.-P.; Gavina, P.; Sauvage, J.-P.; Ventura, B. Rotaxanes Incorporating Two Different Coordinating Units in Their Thread: Synthesis and Electrochemically and Photochemically Induced Molecular Motions. J. Am. Chem. Soc. 1999, 121, 43974408. (e) Bruns, C. J.; Frasconi, M.; Iehl, J.; Hartlieb, K. J.; Schneebeli, S. T.; Cheng, C.; Stupp, S. I.; Stoddart, J. F. Redox Switchable Daisy Chain Rotaxanes Driven by Radical-Radical Interactions. J. Am. Chem. Soc. 2014, 136, 4714-4723. (f) Cnossen, A.; Browne, W. R.; Feringa, B. L. Unidirectional Light-Driven Molecular Motors Based on Overcrowded Alkenes. Top. Curr. Chem. 2014, 354, 139-162.

(3) (a) Sauvage, J.-P. From Chemical Topology to Molecular Machines (Nobel Lecture). Angew. Chem., Int. Ed. 2017, 56, 1108011093. (b) Stoddart, J. F. Mechanically Interlocked Molecules (MIMs)-Molecular Shuttles, Switches, and Machines (Nobel Lecture). Angew. Chem., Int. Ed. 2017, 56, 11094-11125.

(4) (a) Brouwer, A. M.; Frochot, C.; Gatti, F. G.; Leigh, D. A.; Mottier, L.; Paolucci, F.; Roffia, S.; Wurpl, G. W. H. Photoinduction of Fast, Reversible Translational Motion in a Hydrogen-Bonded Molecular Shuttle. Science 2001, 291, 2124-2128. (b) Balzani, V.; Clemente-León, M.; Credi, A.; Ferrer, B.; Venturi, M.; Flood, A. H.; Stoddart, J. F. Autonomous Artificial Nanomotor Powered by Sunlight. Proc. Natl. Acad. Sci. U. S. A. 2006, 103, 1178-1183.

(5) (a) Berrocal, J. A.; Biagini, C.; Mandolini, L.; Di Stefano, S. Coupling of the Decarboxylation of 2-Cyano-2-phenylpropanoic Acid to Large-Amplitude Motions: A Convenient Fuel for an Acid-BaseOperated Molecular Switch. Angew. Chem., Int. Ed. 2016, 55, 69977001. (b) Wilson, M. R.; Solà, J.; Carlone, A.; Goldup, S. M.; Lebrasseur, N.; Leigh, D. A. An Autonomous Chemically Fuelled Small-Molecule Motor. Nature 2016, 534, 235-240. (c) ErbasCakmak, S.; Fielden, S. D. P.; Karaca, U.; Leigh, D. A.; McTernan, C. T.; Tetlow, D. J.; Wilson, M. R. Rotary and Linear Molecular Motors Driven by Pulses of a Chemical Fuel. Science 2017, 358, 340-343. (d) Biagini, C.; Albano, S.; Caruso, R.; Mandolini, L.; Berrocal, J. A.; Di Stefano, S. Variations in the Fuel Structure Control the Rate of the Back and Forth Motions of a Chemically Fuelled Molecular Switch. Chem. Sci. 2018, 9, 181-188. (e) Ghosh, A.; Paul, I.; Adlung, M.; Wickleder, C.; Schmittel, M. Oscillating Emission of [2]Rotaxane 
Driven by Chemical Fuel. Org. Lett. 2018, 20, 1046-1049. (f) Biagini, S.; Di Pietri, F.; Mandolini, L.; Lanzalunga, O.; Di Stefano, S. Photoinduced Release of a Chemical Fuel for Acid-Base-Operated Molecular Machines. Chem. - Eur. J. 2018, 24, 10122-10127. (g) Shi, Q.; Chen, C.-F. Step-by-Step Reaction-Powered Mechanical Motion Triggered by a Chemical Fuel Pulse. Chem. Sci. 2019, 10, 2529-2533.

(6) Bleve, V.; Franchi, P.; Konstanteli, E.; Gualandi, L.; Goldup, S. M.; Mezzina, E.; Lucarini, M. Synthesis and Characterisation of a Paramagnetic [2]Rotaxane Based on a Crown Ether-Like Wheel Incorporating a Nitroxide Motif. Chem. - Eur. J. 2018, 24, 1198-1203.

(7) (a) Martínez-Díaz, M.-V.; Spencer, N.; Stoddart, J. F. The SelfAssembly of a Switchable [2]Rotaxane. Angew. Chem., Int. Ed. Engl. 1997, 36, 1904-1907. (b) Ashton, P. R.; Ballardini, R.; Balzani, V.; Baxter, I.; Credi, A.; Fyfe, M. C. T.; Gandolfi, M. T.; Gómez-López, M.; Martínez-Díaz, M.-V.; Piersanti, A.; Spencer, N.; Stoddart, J. F.; Venturi, M.; White, A. J. P.; Williams, D. J. Acid-Base Controllable Molecular Shuttles. J. Am. Chem. Soc. 1998, 120, 11932-11942.

(8) (a) Sim, B. A.; Milne, P. H.; Griller, D.; Wayner, D. D. M. Thermodynamic Significance of $\rho+$ and $\bar{\rho}$ from Substituent Effects on the Redox Potentials of Arylmethyl Radicals. J. Am. Chem. Soc. 1990, 112, 6635-6638. (b) Wayner, D. D. M.; Sim, B. A.; Dannenberg, J. J. Thermodynamic Properties of Carbocations and Carbanions. Solvation Effects from an Electrochemical and Theoretical (AMI) Study of Some Substituted Benzyl Radicals. J. Org. Chem. 1991, 56, $4853-4858$.

(9) In oxygen-containing media, the measured line width can be expressed as the sum of two terms $W=W_{\text {int }}+b\left[\mathrm{O}_{2}\right]$. One $\left(W_{\text {int }}\right)$ is the intrinsic width and the other the oxygen induced broadening. The latter one is proportional to the oxygen concentration through a constant $b$ which is a function of the interaction radius between oxygen and the radical and of the diffusion coefficient of oxygen in solution.

(10) Pedulli, G. F.; Lucarini, M.; Pedrielli, P.; Sagrini, M.; Cipollone, $\mathrm{M}$. The determination of the oxygen consumption in autoxidation studies by means of EPR spectroscopy. Res. Chem. Intermed. 1996, 22, $1-14$.

(11) A very weak signal of $\mathrm{BPY}^{+\bullet}$ was also detected by EPR after mixing 4 and rotaxane $6 \cdot\left(\mathrm{PF}_{6}\right)_{2}$. The very low intensity of the EPR spectrum of $\mathrm{BPY}^{+}$was attributed to the presence of $\mathrm{O}_{2}$ and reactive radical species in the reaction vessel. Both of them are known to react readily with $\mathrm{BPY}^{+}$to afford diamagnetic products. See: Levey, G.; Ebbesen, T. W. Methyl viologen radical reactions with several oxidizing agents. J. Phys. Chem. 1983, 87, 829-832.

(12) Gualandi, L.; Mezzina, E.; Franchi, P.; Lucarini, M. Nitroxide Radical Spin Probes for Exploring Halogen-Bonding Interactions in Solution. Chem. - Eur. J. 2016, 22, 16017-16021. 